\title{
Mouse iPSC generated with porcine reprogramming factors as a model for studying the effects of non-silenced heterologous transgenes on pluripotency
}

\author{
Petkov $\mathrm{SG}^{1}$, Glage $\mathrm{S}^{2}$, Niemann $\mathrm{H}^{3}$
}

\begin{abstract}
Mouse somatic cells can be reprogrammed to pluripotency by the ectopic expression of four pluripotency transcription factors, Oct4, Sox2, cmyc, and Klf4. Usually, silencing of the exogenous reprogramming factors is considered to be essential for complete reprogramming and differentiation. In the vast majority of studies, murine pluripotency transcription factor sequences have been used for the reprogramming of mouse fibroblasts to induced pluripotent stem cells (iPSC). The effectiveness of xenogeneic transcription factors in miPSC generation has not yet been investigated in detail. Here, we evaluated transposon-based vectors with four porcine pluripotency factors for their ability to reprogram mouse fetal fibroblasts (MEFs) harboring an Oct4-EGFP reporter construct to pluripotency. Additionally, we examined the effects of the non-silenced heterologous transgenes on the expression levels of key endogenous pluripotency markers and the differentiation capacities of the miPSC. Within 8 days of transfection with porcine reprogramming transcription factors the MEFs acquired typical compact miPSC morphology and upregulated expression of endogenous Oct4 and other critical pluripotency genes. Consequently, the transgenes under the control of the TetO promoter became silenced, while the CAG-controlled constructs were expressed throughout the period of culture. Despite the continuous transgene expression, the CAG-miPSC showed normal morphology and were capable of differentiation into the three primary germ layers in vitro and in vivo. However, the expression levels of important endogenous pluripotency markers, Klf4, c-myc, Rex1, and Utf1, were significantly lower in CAG-miPSC compared with TetO-miPSC with silenced reprogramming cassettes. Surprisingly, the endogenous Oct4 and Sox 2 expression levels were not affected by the residual transgene expression. Our results suggest that porcine reprogramming transcription factors are suitable for production of miPSC, but silencing of the heterologous transgenes may be necessary for complete reprogramming to pluripotency.
\end{abstract}

Key Words: iPSC; Reprogramming; Silencing

\section{Introduction}

The fate of a terminally differentiated somatic cell can be reversed by ectopic expression of pluripotency-related factors, as demonstrated by Takahashi and Yamanaka ${ }^{[1]}$. In experiments that have since revolutionized the stem cell field, it was demonstrated that elevated expression of only four proteins such as Oct4, Sox2, c-myc, and Klf4 leads to upregulation of endogenous pluripotency transcription factors and ultimately to acquisition of pluripotent characteristics by the reprogrammed cells ${ }^{[2,3]}$. To underscore the difference to alternative reprogramming methods (e.g. by somatic cell nuclear transfer, cell extracts, etc.), the resulting stem cells have been termed "induced pluripotent stem cells" (iPSC). During the reprogramming process, the balance between the expression levels of the endogenous pluripotency genes and the transgenes is thought to be critical for the success of the reprogramming of somatic cells to iPSC, with the epigenetic silencing of the retroviral transgenes coinciding with the up-regulation of the endogenes ${ }^{[4]}$. It has been shown that over-expression of Oct4 in mouse embryonic stem cells (mESC) leads to mesodermal differentiation $^{[5]}$, while elevated Sox2 expression causes neuroectodermal specification ${ }^{[6]}$. Consequently, the net concentration levels of Oct 4 and Sox 2 in the iPSC would need to be maintained within a narrow range characteristic for ESC. In support of this hypothesis, it was demonstrated that following upregulation of the corresponding endogenous pluripotency factors, the transgenes became gradually silenced in successfully reprogrammed iPS cells ${ }^{[7,8]}$. Persisting transgene expression results in perturbations in the reprogramming process, usually leading to partially reprogrammed states (Class I iPSC), as shown by Mikkelsen and co-workers ${ }^{[9]}$. Residual transgene expression restricted the differentiation capacities of human iPSC ${ }^{[10]}$ and affected epigenetic signatures and gene expression levels of miPSC compared with $\mathrm{mESC}^{[11]}$. Moreover, in a recent report, miPSC produced with transposon-delivered transgenes that were continuously expressed by supplementation with doxycycline (DOX) were reversed to an alternative pluripotency state (F-state), characterized by aberrant colony morphology and dependency on the continuous transgene expression for their maintenance ${ }^{[12]}$.

It has been convincingly demonstrated that the differentiation capacities of murine iPSC lines with constitutive expression of nonsilenced reprogramming factors are limited. However, conflicting results were reported by different research groups. The miPSC produced in the first attempts of Takahashi and Yamanaka ${ }^{[1]}$ did not silence the reprogramming factors and were not able to differentiate

Author Names in full: Stoyan G Petkov ${ }^{1}$, Silke Glage ${ }^{2}$, Heiner Niemann ${ }^{3}$

${ }^{1}$ German Primate Center, Goettingen, Germany, ${ }^{2}$ Hannover Medical School, Hannover, Germany, ${ }^{3}$ Institute for Farm Animal Genetics (FLI), Neustadt, Germany 
and form chimeras. Similarly, transgene-expressing miPSC were unable to differentiate and upon injection into immunodefficient mice formed homogenous tumors, consisting of undifferentiated cells expressing high levels of Oct $4^{[13]}$. On the other hand, the use of a constitutively expressed reprogramming cassette led to miPSC with normal ESC-like characteristics, which were capable of forming the three primary germ layers in teratomas ${ }^{[14]}$. However, these cells also exhibited gene expression differences compared with ESC and excision of the loxP-flanked transgenes resulted in miPSC with complete pluripotency characteristics.

While the vast majority of miPSC described in the literature have been generated with murine pluripotency transcription factors, there have been very few reports on the use of transgenes from other species. Only one study has reported the use of human reprogramming factors to successfully reprogram mouse fibroblasts to iPSC $^{[15]}$. In addition, the silencing of non-murine reprogramming factors as well as the effects of the non-silenced xenogeneic transgenes on the expression of endogenous pluripotency genes and its relationship with differentiation of miPSC have not been yet investigated. Here, we explored the possibility to use porcine transcription factors for the reprogramming of mouse embryonic fibroblasts (MEFs) to pluripotency. Established miPSC lines constitutively expressing the four porcine Yamanaka factors (pOCT4, pSOX2, pc-MYC, and pKLF4) under the control of the ubiquitous CAG promoter were characterized for pluripotency and their differentiation potential together with the expression levels of master pluripotency genes were compared with those of miPSC produced either with a silenced or CRE recombinase-excised reprogramming cassette.

\section{Materials and methods}

Unless specified otherwise, the chemicals and kits used in this study were purchased from Sigma-Andrich.

Derivation of mouse fetal fibroblasts for feeder cells and reprogramming

Outbred NMRI mice were purchased from Harlan Laboratories (Horst, Netherlands) and were used to establish MEFs for feeder layers. C57BL/6 mice harboring Oct4-EGFP pluripotency gene reporter (OG2) were kindly provided by Prof. Hans Schoeler (MaxPlanck-Institute for Molecular Biomedicine, Muenster, Germany) and were used to generate MEFs for reprogramming to iPSC. For the generation of MEFs, pregnant mice were sacrificed at day 13-13.5 of gestation, fetuses were dissected and the heads, livers, mesonephros, and hearts were removed. The remaining parts, including limb buds and body wall were minced with a scalpel blade, incubated in trypsin/EDTA solution for $20 \mathrm{~min}$ at $37^{\circ} \mathrm{C}$ and disaggregated to single cells and clumps by vigorous pipetting after adding warm culture medium. The culture medium used was high glucose DMEM (Lonza), supplemented with $15 \%$ fetal bovine serum (FBS) (Gibco, Lot 41Q2035K), non-essential amino acids (GE Healthcare), sodium pyruvate, $2 \mathrm{mM}$ Glutamine (GE Healthcare), and $0.1 \mathrm{mM} \mathrm{2-}$ mercaptoethanol. Feeder cells were produced by treating MEFs from NMRI mice with $10 \mu \mathrm{g} / \mathrm{ml}$ mitomycin $\mathrm{C}$ for 2 hours.

\section{Reprogramming expression vectors}

The construction of the Sleeping Beauty (SB) transposon plasmids with either the Tet-On (TetO) promoter (SB-TetO-pOSMK-IRESTomato, used together with SB-CMV-rTA-IRES-Neo ${ }^{r}$ ) or with the constitutive CAG promoter (SB-CAG-pOSMK-IRES-Tomato) for expression of the porcine OCT4, SOX2, c-MYC, and KLF4 have been described previously ${ }^{[16]}$. To create a CRE-excisable version, the CAG promoter and the polyadenylation signal were amplified using loxP-containing forward or reverse primer, respectively, and these modified sequences were used to replace the originals by standard DNA cloning techniques, thus generating a loxP-flanked CAGpOSMK-IRES-Tomato-pA cassette. For efficient integration into MEFs, the plasmid with a hyperactive version of the SB (SB100x) was co-transfected with each of the reprogramming transposons. The SB transposon vector backbone and transposase expression plasmids were provided by Dr. Zoltan Ivics (Paul-Ehrlich-Institute, Langen, Germany).

\section{Mouse iPSC generation and culture}

Three different MEF lines from C57BL/6-OG2 mice were electroporated at passages 3-4 with the SB transposon and transposase plasmids as described by Petkov et al. ${ }^{[16]}$ and plated on gelatinized T75 flasks. The cells were cultured in MEFs culture medium for 2-3 days and then were then split 1:3-1:4 to T75 flasks with mitotically inactivated MEF feeders and the medium was supplemented with $1000 \mathrm{U} / \mathrm{ml}$ ESGRO murine leukemia inhibitory factor (mLIF) (Millipore). When vectors with the TetO promoter were used, the medium was supplemented with $5 \mu \mathrm{g} / \mathrm{ml}$ DOX. The OCT4-EGFP-positive colonies with ESC-like morphology were manually picked at day 10-11 post-transfection, disaggregated with Trypsin/EDTA, and cultured individually on mytomycin Cinactivated MEFs to establish individual cell lines. The established miPSC were regularly split every 3-4 days at ratio $1: 15-1: 20$ by trypsinization and maintained on fresh feeders in ESGRO medium for over 70 passages. For generation of transgene-free miPSC, the miPSC harboring the "floxed" version of the reprogramming transposon were transfected with SB-CAG-CRE-IRES-Puro ${ }^{\mathrm{r}}$ vector using Lipofectamine 2000 (Life Sciences) as instructed by the manufacturer and selected with culture medium supplemented with 3 $\mu \mathrm{g} / \mathrm{ml}$ puromycin.

\section{Alkaline phosphatase (AP) staining and immunocytochemistry}

The cultured were fixed with $4 \%$ Formalin solution for 5 min (for $\mathrm{AP}$ ) or $15 \mathrm{~min}$ (antibody staining) and washed with PBS prior to further processing. For AP staining, a solution containing $1 \mathrm{mg} / \mathrm{ml}$ Fast Red and $0.4 \mathrm{mg} / \mathrm{ml}$ Naphtol-BI-Phosphate in AP-buffer (100 $\mathrm{mM} \mathrm{NaCl} 2,100 \mathrm{mM}$ Tris ( $\mathrm{pH} 9.5$ ), and $50 \mathrm{mM} \mathrm{MgCl}_{2}$ ) was applied to the fixed wells. The AP-positive cells were discerned by the formation of insoluble red-brown precipitate. The presence of SSEA1, SSEA-3, SSEA-4, TRA-1-60, and TRA-1-81 expression in miPSC was assessed by incubation with primary antibodies (Thermo; Cat. numbers 41-1200, MA1-020X, 41-4000, 41-1000, 41-1100, respectively), diluted in PBS with 2\% Knockout Serum Replacement (KSR) (Invitrogen) at concentration $10 \mathrm{ng} / \mathrm{ml}$ for 1 hour, followed by washing with PBS and incubation with the secondary peroxidase conjugated antibody. For staining of neuronal-like cells, the anti-PGP 9.5 primary antibody (DACO) was used in a similar protocol. The expressed proteins were visualized after incubation with peroxidase substrate from AEC staining kit.

\section{Reverse transcription, PCR, and real-time PCR}

For RNA extraction, the miPSC cultures were washed 3 times with $\mathrm{Ca}-$ and $\mathrm{Mg}$-free PBS and incubated in the same solution for 15 minutes at 37 degrees $\mathrm{C}$. The miPSC colonies were detached from the feeders by gentle pipetting, collected in a $15 \mathrm{ml}$ centrifuge tube and allowed to settle on the bottom of the tube for $10 \mathrm{~min}$. The supernatant containing single cells (presumably mostly feeder MEFs) was carefully removed, the pellet was re-suspended in PBS and the colonies were pelleted by centrifugation, lysed in TRI reagent (Ambion), and total RNA was extracted as instructed by the manufacturer. Reverse-transcription was carried out with MuLV Reverse Transcriptase (Applied Biosystems). Polymerase chain reaction was performed using $50 \mathrm{ng}$ reverse transcribed RNA per $25 \mu \mathrm{l}$ reaction volume using Platinum Taq Polymerase (Invitrogen). 
Real-time relative quantitative analysis was run on the ABI 7500 Fast System using SYBR Green Master Mix (Applied Biosystems). The primers used for PCR and real-time PCR analysis of endogenous mouse pluripotency markers Oct4, Sox2, Nanog, c-myc, Klf4, Rex1, and Utf1 as well as for the differentiation markers alpha fetoprotein (Afp), Sox17, Gata4, Gata6, cardiac troponin, myosin heavy chain (MHC), nestin, $\beta$-III tubulin, and Pax6 are shown in Table S1. The primers used for the amplification of the mouse endogenous pluripotency genes were specific only for the mouse cDNA sequences and did not amplify the pig pOSMK cassette (results not shown). The primers used for the amplification of the pOSMK-IRES-Tomato construct were a forward primer binding at the end of KLF4 and a reverse primer binding to IRES (shown in Table S1). The data from the relative quantitative analysis were analyzed with GeneEx software (bioMCC). Statistically significant differences were determined by Student's t-test performed by the same computer program.

\section{Karyotyping}

Three lines each from TetO- and CAG miPSC at passages 58-60 were treated with Demecolcine solution (Sigma, D1925) added at $100 \mu \mathrm{l} / 10 \mathrm{ml}$ culture medium for $35 \mathrm{~min}$. The cells were harvested by trypsinization, incubated in hypotonic solution $(0.28 \% \mathrm{KCl}+0.25 \%$ $\mathrm{Na}$ Citrate) for $25 \mathrm{~min}$ at $37^{\circ} \mathrm{C}$, and fixed in cold $\left(-20^{\circ} \mathrm{C}\right)$ methanol: acetic acid (3:1) fixative for 1 hour. Metaphase spreads were produced by dropping $50 \mu \mathrm{l}$ drops onto glass slides placed at a slight angle over steaming water bath $\left(95^{\circ} \mathrm{C}\right)$ and waiting 1 min before removal and air drying. The metaphase spreads were stained with $2 \%$ Giemsa solution. The metaphase chromosomes of at least 15 metaphase spreads per cell line were counted to determine whether the cells had normal karyotype.

\section{Embryoid body (EB) formation}

Mouse iPSC colonies were separated from the feeder as described above in subsection 2.5, disaggregated to single cells with trypsin, and suspended in basic culture medium without ESGRO. "Hanging drops" were then produced by making $25 \mu \mathrm{l}$ drops on the inner side of a

Phase-contrast
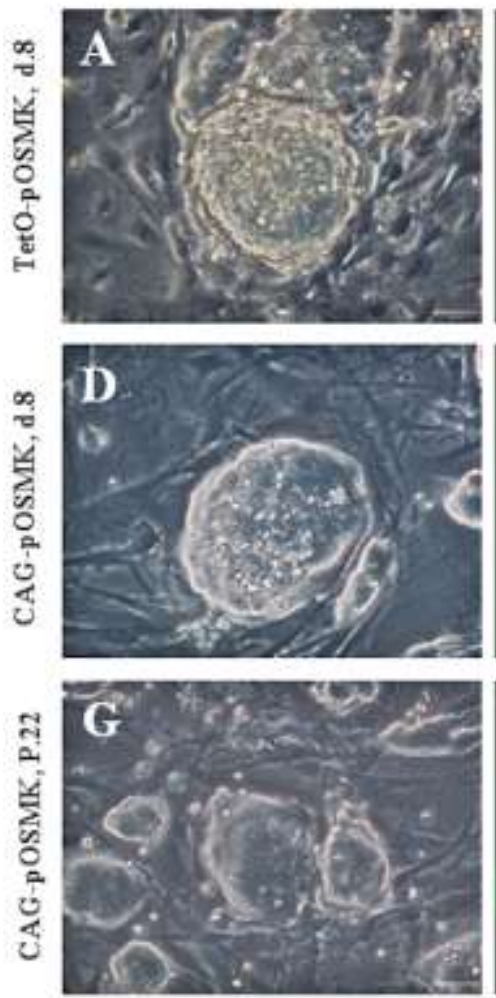

UV-EGFP
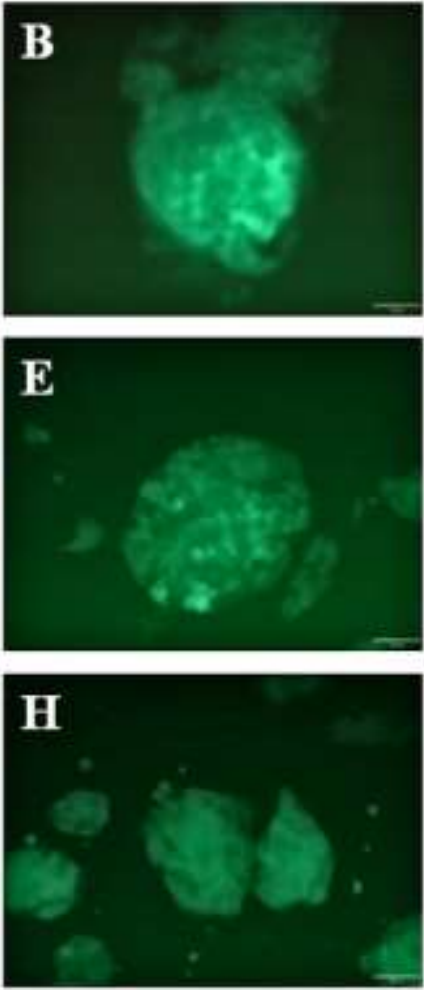

$10 \mathrm{~mm}$ Petri dish lid (containing 2000 cells/drop) and placing the lid back on the dish filled with PBS (to prevent drying of the drops). After 5 days in "hanging drops" culture, the emerging embryoid bodies were plated in gelatin-treated 6-well plates and cultured further for 15 days with changes of the medium every 3-4 days. For neuronal differentiation, the attached EBs were cultured in N2 medium (DMEM/F12 supplemented with sodium pyruvate, amino acids, penicillin-streptomycin, and N2 supplement (Thermo)).

\section{Teratoma formation}

Prior to conducting the animal experiments, permission was obtained from the local animal welfare authority (LAVES).

Nude immunodeficient mice were produced by mating outbred NMRI strain parents. A total of 13 nude mice ( 7 mice/TetO group and 6 mice/CAG group) were injected each with $1 \times 10^{6}$ putative iPSC derived from reprogramming with the porcine vectors subcutaneously in the right flank. The mice were observed daily for 3 weeks. After reaching the critical size of the tumour $(1 \mathrm{~cm})$, the animals were sacrificed. Biopsies were taken for DNA/RNA extraction, and tissues were fixed in neutral buffered $4 \%$ formalin not exceeding $48 \mathrm{~h}$, dehydrated (Shandon Hypercenter, XP) and subsequently embedded in paraffin (TES, Medite). Sections (2-3 $\mu \mathrm{m}$ thick, microtome Reichert-Jung 2030), were deparaffinized in xylene and H\&E stained according to standard protocols. The morphological evaluation for the presence of the three germ layers (Axioskop 40, Zeiss microscope) was performed by a trained pathologist and representative microphotographs were taken (AxioCam MRc, Zeiss).

\section{Results}

\section{Reprogramming to pluripotency}

Transfected MEFs cultured on inactivated feeders formed O4-EGFPpositive colonies with typical miPSC-like morphology within 7-8 days post-transfection (Figure 1A, D). A number of granular colonies consisting of round, highly refractory cells were also observed.

\section{UV-Tomato}
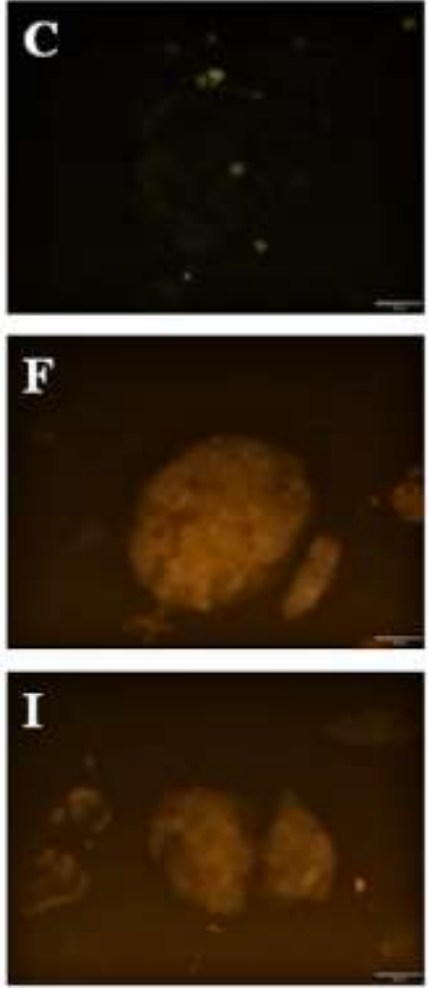

Figure 1. Mouse iPSC generated with Sleeping Beauty transposons carrying porcine reprogramming factors. A) TetO-miPSC at day 8 post-transfection with typical miPSC morphology. B) Oct4-EGFP reporter expression in the IRES-Tomato expression except in some cells in image A. D) CAG-miPSC at day 8 post-transfection. The colony has compact miPSC-like morphology. E) Expression of Oct4-EGFP reporter in the colony shown in image $D$. F) Expression of non-silenced pOSMKIRES-Tomato construct in the colony from image D. G) CAG-miPSC at P.22. The colonies maintained compact miPSC morphology. H) Oct4-EGFP reporter expression in the colonies shown in image G. I) Expression of pOSMK-IRESTomato in the colonies shown in image G. (All scale bars $=50 \mu \mathrm{m})$. colony in image A. C) Silenced pOSMK- 
There was no significant difference in the timing of EGFP-positive colony appearance between the different groups where transposons with TetO or CAG were used. In most miPSC colonies produced with the TetO promoter, the Tomato fluorescence (indicating pOSMK transgene expression) disappeared completely from the cells by day 10-11 of primary cultures (Figure 1C), and was no longer detectable in the presence of DOX, indicating successful transgene silencing. The colonies that retained the Tomato fluorescence were not picked. All miPSC produced with CAG promoters retained weak Tomato expression throughout the entire time of culture (>70 passages) (Figure $1 \mathrm{~F}, \mathrm{I})$. All of the picked colonies (12 colonies/transfected MEF line) formed clonal lines when cultured in individual wells; however, some TetO-lines showed signs of differentiation after 3-4 passages and were discarded. Five lines from each group were chosen for long-term culture.

\section{Expression of master pluripotency markers}

The pluripotency-related surface antigens AP and SSEA-1 were expressed in both groups, while SSEA-3, SSEA-4, TRA-1-61, and TRA-1-80 were not detected (Figure 2). RT-PCR confirmed expression of the endogenous Oct4, Sox2, Nanog, c-myc, Klf4, Rex1, and Utf1 in both CAG- and TetO-miPSC (Figure 3A). Expression of the transgenes was detected only in the CAG group (Figure 3A). To determine whether there was a difference in the expression levels of endogenous pluripotency genes between the CAG and TetO groups, we carried out real-time relative quantitation analysis. While there was no significant difference in the expression of Oct4, Sox2, and Nanog $(\mathrm{P}>0.05)$, the expression levels of c-myc, Klf4, Rex1, and Utf1were significantly higher in the TetO group compared with the CAG-miPSC $(\mathrm{p}<0.05)$ (Figure 3B). When the pOSMK cassette was removed from the CAG group by CRE expression, there was no significant change in the expression levels of the endogenous pluripotency genes ( $p>0.05)$, except for Utf1, which was up-regulated in the CRE-treated cells $(\mathrm{p}<0.01)$ (Figure 3C).

\section{Karyotyping}

Each of the 6 examined miPSC lines had 20 chromosome pairs and no aberrations from the normal diploid karyotype were found (Figure S1).

\section{In vitro and in vivo differentiation}

The cells from both experimental groups formed EBs within 5 days of differentiation culture in "hanging drops". Upon attachment on gelatin-treated plastic surfaces, the cells expanded and spontaneously differentiated into epithelial-like cells (Figure 4A, endoderm), neuronal-like cells that reacted positively with the anti-PGP 9.5 antibody (Figure 4A, ectoderm), and rhythmically contracting cardiac myocytes (Figure 4A, mesoderm; Video S1). The expression of differentiation markers Afp, Sox17, Gata4, Gata6, MHC, troponin, nestin, $\beta$-III tubulin, and Pax6 in EB was confirmed by RT-PCR (Figure 4B).

When injected into immunodefficient mice, $3 / 7$ (43\%) mice injected with cells from the TetO group and 6/6 (100\%) mice injected with miPSC from the CAG group formed teratomas within 3 weeks postinjection. Histological analysis of the teratomas revealed presence of the three germ layers in teratomas produced with miPSC from both the TetO and CAG groups (Fig. 5). We also visually examined fresh biopsies for the presence of EGFP fluorescence as indication of undifferentiated cells and found EGFP-positive cells in one of the CAG-miPSC-derived teratomas. The expression levels of key endogenous pluripotency genes, the pOSMK transgenes, and main differentiation markers were compared between miPSC from the CAG group and the teratomas by real-time relative quantitative analysis. As expected, Oct4, Nanog, Rex1, and Utf1 were significantly down-regulated, while differentiation markers Afp, Sox17, Gata4, Gata6, Pax6, and Nestin were significantly upregulated in teratomas (all p<0.01) (Fig. 6). There was no significant difference in the expression levels of Sox 2 and Klf4, while c-myc was significantly higher expressed in teratomas $(\mathrm{P}<0.05)$. These results were similar to the TetO group, except for $\mathrm{c}-\mathrm{myc}$, which did not show a significant difference (results not shown). Expression of the reprogramming pOSMK cassette could still be detected in two teratomas generated with CAG-miPSC, but the expression levels were low, as suggested by real-time PCR threshold values higher than 33 , so relative quantitative analysis could not be reliably completed.
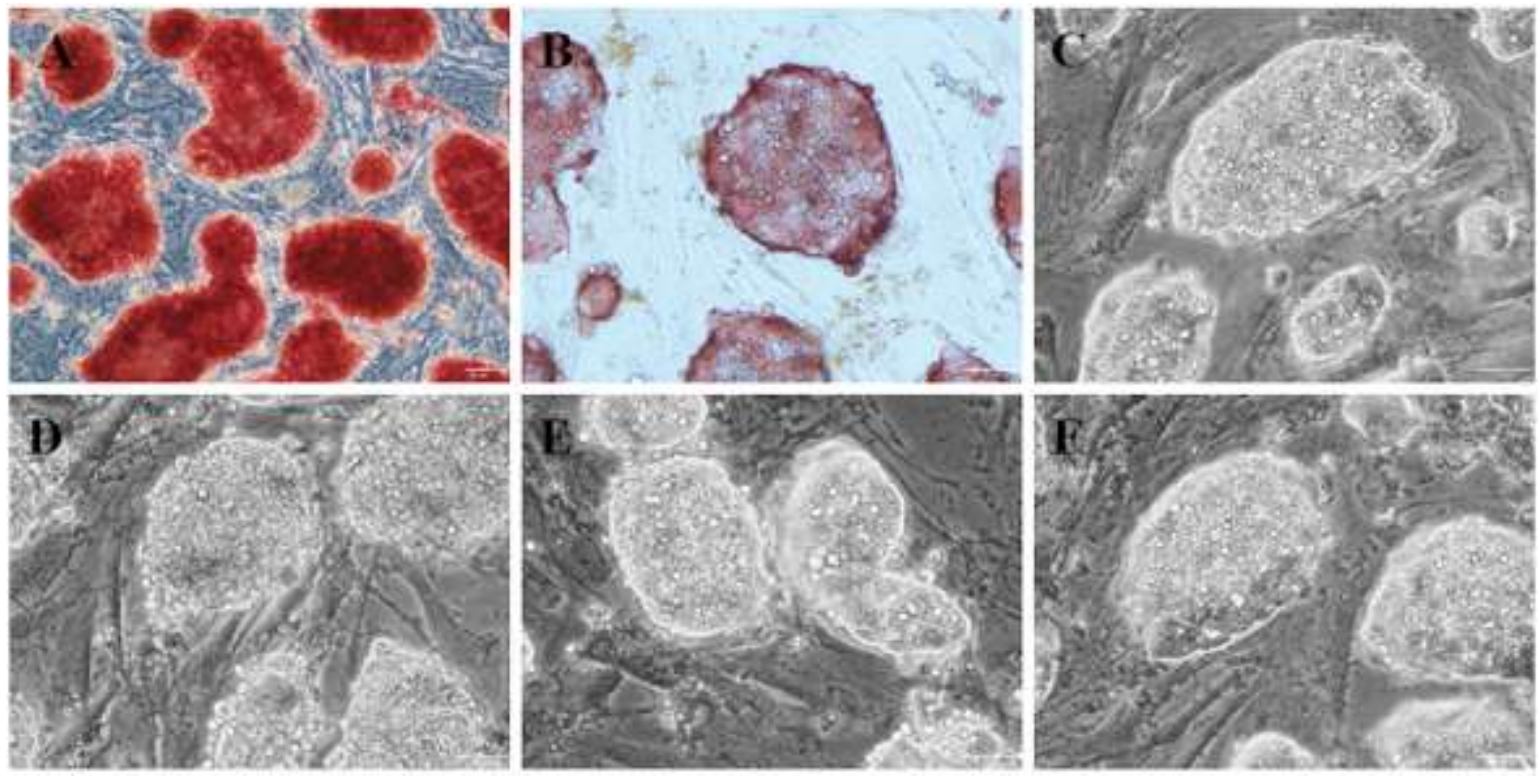

Figure 2. Expression of surface pluripotency markers in CAG-miPSC at P. 22. A) AP. B) SSEA-1. C) SSEA-3. D) SSEA-4 E) TRA-1-60. F) TRA-1-81. (Scale bars = $50 \mu \mathrm{m})$. 
A

RT-PCR of endogenous pluripotency genes

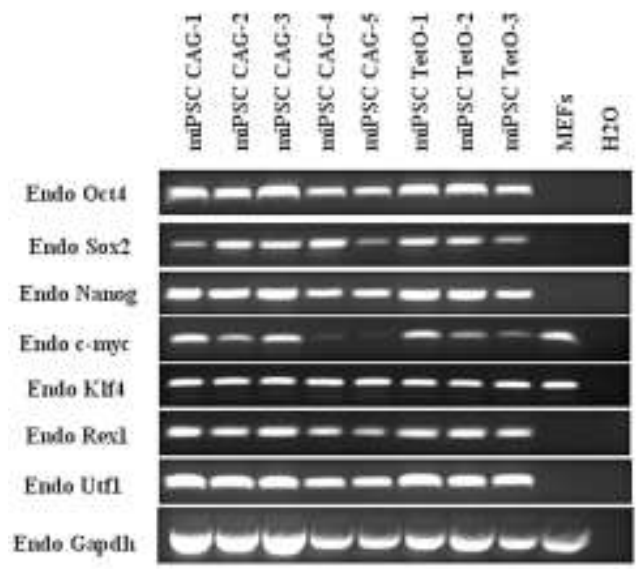

RT-PCR of porcine reprogramming factors

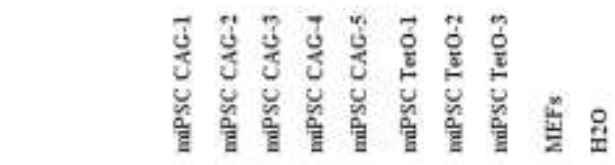

tans-pOSMK
B

Real-time relative quantitatien PCR

Relativeplurpotency gene enpresdat in miPSC

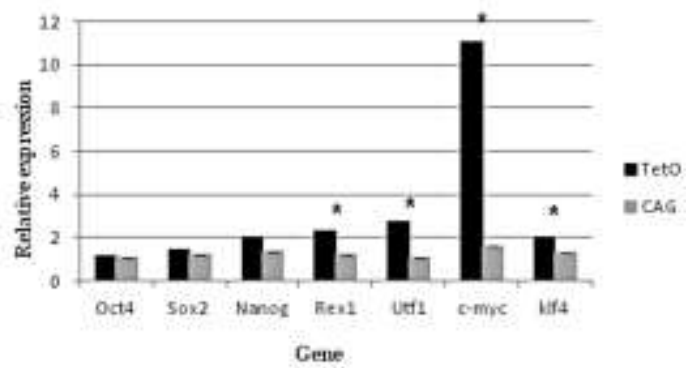

$\mathrm{C}$

Relative phripotency gene expression in miPSC with and without pOSMIK

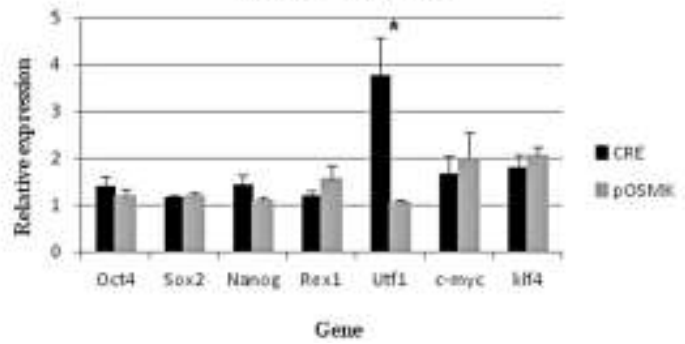

Figure 3. Gene expression in miPSC produced with porcine reprogramming factors. A) RT-PCR analysis of major endogenous pluripotency genes and transgenes in miPSC. B) Real-time relative quantitative analysis of expression level differences between TetO-and CAG-miPSC. C) Real-time relative quantitative analysis of expression level differences between miPSC with CRE-excised and still expressed transgenes. Statistically significant differences ( $p<0.05$ ) are indicated with asterisks.

A

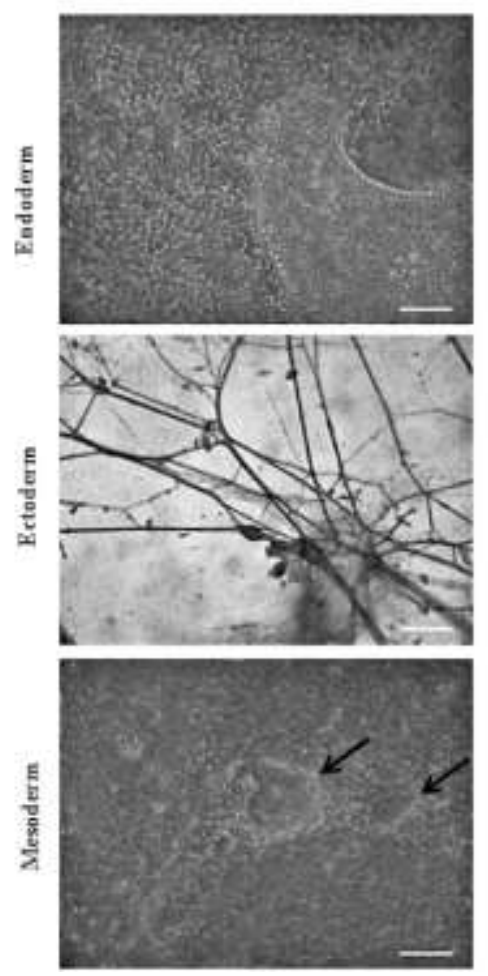

B

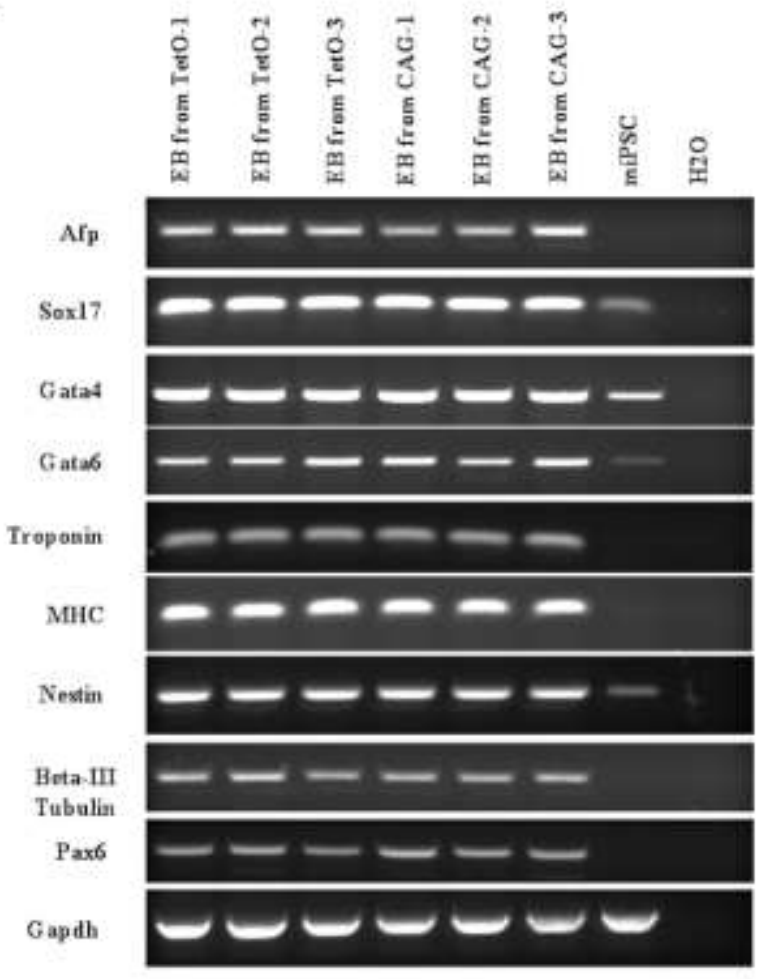

Figure 4. In vitro and in vivo differentiation of miPSC produced with porcine reprogramming factors. A) In vitro differentiation of CAG-miPSC: epithelial-like cells (endoderm) (scale bar $=50 \mathrm{~mm}$ ), neuronal-like cells positive for PGP 9.5 (ectoderm) (scale bar = 20 mm), and spontaneously contracting cardiac myocytes (mesoderm), indicated with arrows (scale bar $=50 \mathrm{~mm}$ ). (For additional data on cardiomyocyte contraction, see Supplementary Video 1). B) Expression of differentiation markers in EB outgrowths by RT-PCR. 
Endoderm
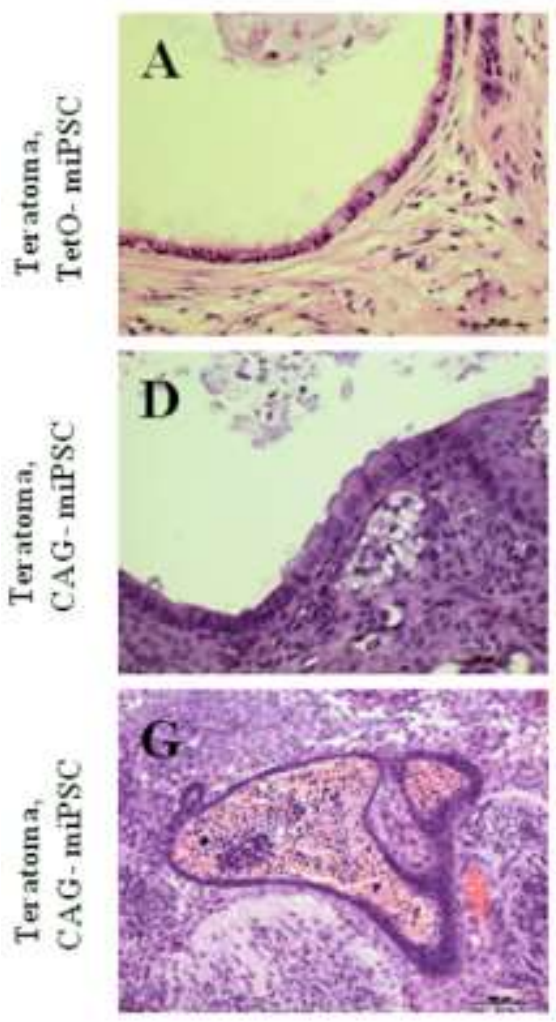

Ectoderm
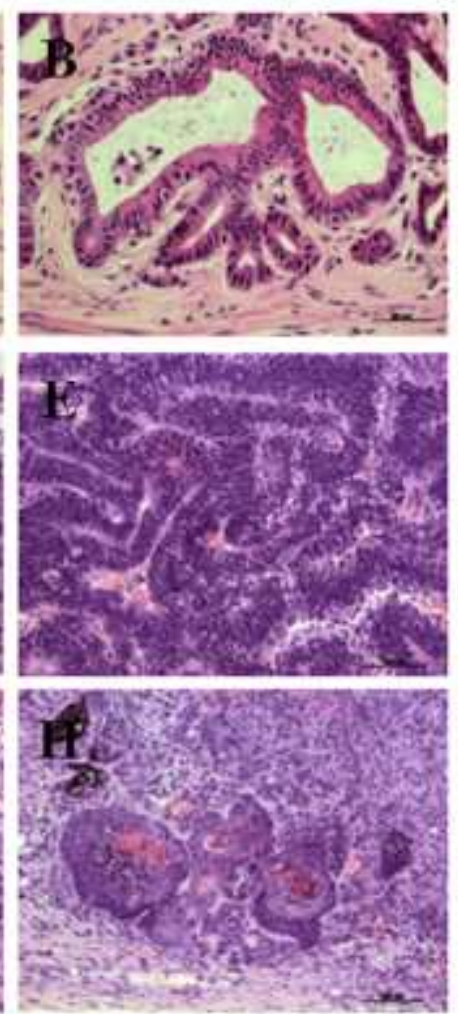

Mesoderm
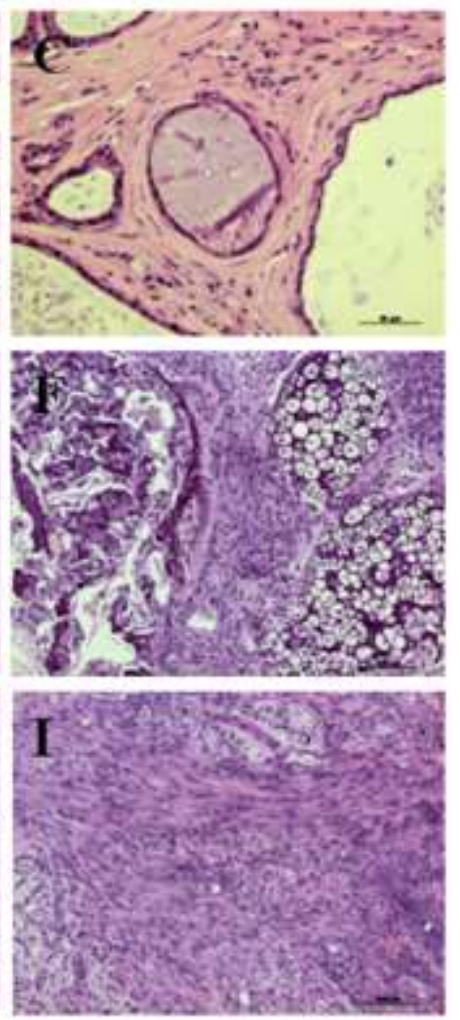

Figure 5. In vivo differentiation of miPSC in teratomas. TetO-miPSC injected into nude mice formed ciliated epithelium with mucous- producing cells (A), neuronal tubes $(B)$, and connective tissue $(C)$ (scale bars $=50 \mu \mathrm{m})$. CAG-miPSC differentiated into ciliated epithelium $(D)$, neural tubes $(E)$, cartilage and bone $(F)$, epithelial cysts $(G)$, keratinized epithelium $(H)$, and connective tissue $(I)$. (Scale bars $=100 \mu \mathrm{m})$.

Relative gene expression in mouse iPSC and teratomas

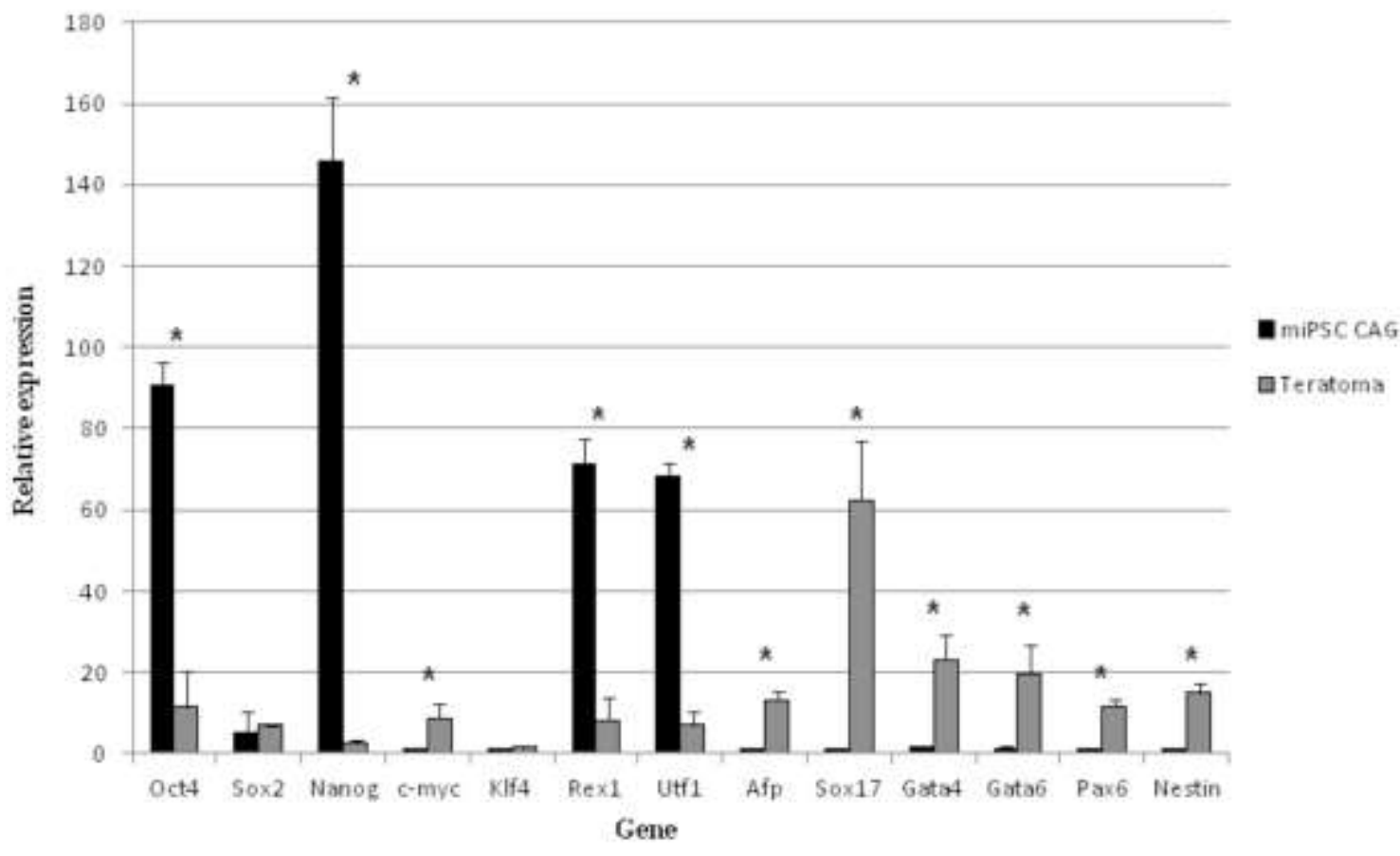

Figure 6. Real-time relative quantitation analysis of gene expression in CAG-miPSC and teratomas. Statistically significant differences are indicated with asterisks. 


\section{Discussion}

Here, we investigated whether four porcine reprogramming transcription factors were able to successfully reprogram MEFs to pluripotency. The amino acid sequences for Oct4, Sox2, c-myc, and Klf4 are evolutionary highly conserved, although not fully identical between mouse and pig (88\%, 98\%, 93\%, and 94\%, respectively; amino acid sequence alignments are shown on Figure S2). Thus, it was necessary to confirm that the porcine proteins can in fact reprogram somatic cells across species. As expected, the MEFs changed proliferation and morphology and upregulated endogenous OCT4 expression, as demonstrated by the presence of EGFP fluorescence and subsequently confirmed by RT-PCR. The picked colonies proliferated robustly and established cell lines with typical compact miPSC morphology and expression of key pluripotency markers such as AP, SSEA-1, Oct4, Sox2, Nanog, Klf4, Rex1, and Utf1. At the same time, the TetO-promoter-controlled transgenes were silenced, even in the presence of DOX. This result is in contrast with other reports, where transposon-delivered transgenes were not silenced by the reprogrammed cells if the culture medium was continuously supplemented with $\mathrm{DOX}^{[12,17]}$. A possible explanation for our results is that the porcine transgenes are more efficiently recognized and silenced by the miPSC. Silencing of the reprogramming transcription factors has been described as an essential prerequisite for successful reprogramming[2, 7- 9]. Thus, porcine reprogramming factors may be useful for the production of miPSC, particularly where significant nucleotide sequence differences between the transgenes and the endogenous pluripotency genes are desired.

Another interesting question of this study was whether the mouse cells would balance the expression levels of key endogenous pluripotency genes with the non-silenced porcine transgenes. We obtained cell lines with silenced (TetO-regulated) and non-silenced (CAG-regulated) reprogramming cassettes, which enabled us to address this question by comparing the expression levels of endogenous pluripotency genes between the two groups. Surprisingly, we found no significant differences in the expression levels of endogenous mouse Oct 4 and Sox 2 between the cells with silenced vs. expressed transgenes. Additionally, the expression levels of these genes did not change even after the transgenes were removed by expression of CRE recombinase. These results suggest that the porcine transgenes did not participate in the regulation of endogenous Oct 4 and Sox 2 expression after the initial induction. Moreover, none of the cells from the CAG-group showed any signs of differentiation that would be expected from elevated total Oct 4 and Sox 2 expression levels. A possible explanation for this outcome could be the very low expression levels of the transgenes, as suggested by our real-time PCR analysis. Another possibility is that the specifying signals ensuing from elevated total Oct 4 and Sox 2 levels had balanced each other, preventing differentiation into any of the germ layers. On the other hand, the expression levels of the endogenous c-myc, Klf4, Rex1, and Utf1 were significantly lower in cell lines with nonsilenced transgenes compared with the TetO group. Excision of the reprogramming cassette by CRE-recombinase resulted in significant upregulation of Utf1, but failed to upregulate the rest.

Despite the deviations in the expression of important pluripotency markers, the cell lines with residual expression of the four Yamanaka factors were capable of differentiation into derivatives of the three primary germ layers in vitro and in vivo (by forming fully differentiated teratomas) similar to the TetO-group. When we examined the expression levels of pluripotency and differentiationrelated genes in teratomas and iPSC lines, we found that, as expected, the pluripotency markers were down-regulated while the differentiation markers were up-regulated, suggesting normal differentiation process in the CAG-iPSC.
Unlike the miPSC in an alternative state of pluripotency (F-state) described recently ${ }^{[12]}$, our miPSC lines with non-silenced reprogramming factors have compact ES-like morphology and do not depend on the expression of the transgenes for their maintenance; therefore, they do not necessarily fall into an alternative pluripotency state category. Nevertheless, it has been shown that even subtle aberrations in miPSC with residual transgene expression could impair the full developmental competency of miPSC ${ }^{[11,14]}$. The lower expression of important pluripotency markers such as Rex1, Utf1, and Klf4 in CAG-miPSC suggests that this may also apply for miPSC produced with non-silenced porcine transcription factors. In future work, the cells will be tested for chimera formation to ascertain whether they are fully competent iPSC.

\section{Conclusions}

In conclusion, we successfully established miPSC using four porcine reprogramming transcription factors and examined the effects of the non-silenced transgenes on the expression levels of key endogenous pluripotency markers. The residual expression of the transgenes affected expression levels of important pluripotency genes such as cmyc, Klf4, Rex1, and Utf1. These results suggest that porcine pluripotency transcription factors are suitable for reprogramming of mouse somatic cells to pluripotency, but epigenetic silencing of the xenogeneic transgenes may be necessary for complete acquisition of pluripotency.

\section{References}

1. Takahashi K. and Yamanaka S. Induction of pluripotent stem cells from mouse embryonic and adult fibroblast cultures by defined factors. Cell 2006; 126(4): 663-76.

2. Okita K, Ichisaka $\mathrm{T}$, Yamanaka S. Generation of germlinecompetent induced pluripotent stem cells. Nature 2007; 448: 3137.

3. Takahashi K, Tanabe K, Ohnuki M, Narita M, Ichisaka T, Tomoda $\mathrm{K}$, Yamanaka S. Induction of pluripotent stem cells from adult human fibroblasts by defined factors. Cell 2007; 131(5): 861-72.

4. Hotta A and Ellis J. Retroviral Vector Silencing During iPS Cell Induction: An Epigenetic Beacon that Signals Distinct Pluripotent States. J Cell Bioch. 2008; 105(4): 940-8.

5. Niwa H, Miyazaki J, Smith A. Quantitative expression of Oct-3/4 defines differentiation, dedifferentiation or self-renewal of ES cells. Nat Genet 2000; 24(4): 372-6.

6. Kopp J, Ormsbee B, Desler M, Rizzino A. Small increases in the level of Sox 2 trigger the differentiation of mouse embryonic stem cells. Stem Cells 2008; 26(4): 903-11.

7. Papapetrou E, Tomishima M, Chambers S, Mica Y, Reed E, Menon J, Tabar V, Mo Q, Studer L, Sadelain M. Stoichiometric and temporal requirements of Oct4, Sox2, Klf4, and c-Myc expression for efficient human iPSC induction and differentiation. 2009; 106(31): 12759-64.

8. Wernig $\mathrm{M}$, Meissner $\mathrm{A}$, Foreman $\mathrm{R}$, Brambrink $\mathrm{T}, \mathrm{Ku} \mathrm{M}$, Hochedlinger $\mathrm{K}$, Bernstein BE, Jaenish R. In vitro reprogramming of fibroblasts into a pluripotent ES-cell-like state. Nature 2007; 448(7151): 318-24.

9. Mikkelsen T, Hanna J, Zhang X, Ku M, Wernig M, Schorderet P, Bernstein BE, Jaenish R, Lander ES, Meissner A. Dissecting direct reprogramming through integrative genomic analysis. Nature 2008; 454(7200): 49-55. 
10. Ramos-Mejía V, Montes R, Bueno C, Ayllón V, Real P, Rodríguez $\mathrm{R}$, Menendez P. Residual expression of the reprogramming factors prevents differentiation of iPSC generated from human fibroblasts and cord blood CD34+ progenitors. PLoS One 2012; 7(4): e35824.

11. Sommer C, Christodoulou C, Gianotti-Sommer A, Shen S, Sailaja B, Hezroni H, Spira A, Meshorer E, Kotton DN, Mostoslavsky G. Residual expression of reprogramming factors affects the transcriptional program and epigenetic signatures of induced pluripotent stem cells. PLoS One 2012; 7(12): e51711.

12. Tonge PD, Corso AJ, Monetti C, Hussein SM, Puri MS, Michael IP, Li M, Lee DS, Mar JC, Cloonan N, Wood DL, Gauthier ME, Korn O, Clancy JL, Preiss T, Grimmond SM, Shin JY, Seo JS, Wells CA, Rogers IM, Nagy A. Divergent reprogramming routes lead to alternative stem-cell states. Nature 2014; 516(7530), 192-7.

13. Brambrink T, Foreman R, Welstead G, Lengner C, Wernig M, Suh $\mathrm{H}$, Jaenish R. Sequential expression of pluripotency markers during direct reprogramming of mouse somatic cells. Cell Stem Cell 2008; 2(2): 151-9.
14. Sommer CA, Sommer AG, Longmire TA, Christodoulou C, Thomas DD, Gostissa M, Alt FW, Murphy GJ, Kotton DN, Mostoslavsky G. Excision of reprogramming transgenes improves the differentiation potential of iPS cells generated with a single excisable vector. Stem Cells 2010; 28(1): 64-74.

15. Talluri T, Kumar D, Glage S, Garrels W, Ivics Z, Debowski K, Behr R, Kues W. Non-viral reprogramming of fibroblasts into induced pluripotent stem cells by Sleeping Beauty and piggyBac transposons. Bioch Bioph Res Com. 2014; 450(1), 581-7.

16. Petkov S, Hyttel P, Niemann $H$. The choice of expression vector promoter is an important factor in the reprogramming of porcine fibroblasts into induced pluripotent cells. Cell Repr. 2013; 15(1): $1-8$.

17. Woltjen K, Michael IP, Mohseni P, Desai R, Mileikovsky M, Hämäläinen R, Cowling R, Wang W, Liu P, Gertsenstein M, Kaji K, Sung HK, Nagy A. PiggyBac transposition reprograms fibroblasts to induced pluripotent stem cells. Nature 2009; 458(7239): 766-70.

\author{
Abbreviations \\ Afp: Alpha-fetoprotein \\ AP: Alkaline Phosphatase \\ DMEM: Dulbecco's Modified Eagle's Medium \\ DOX: Doxycycline \\ EGFP: Enhanced Green Fluorescent Protein \\ FBS: Fetal Bovine Serum \\ iPSC: Induced Pluripotent Stem Cells \\ mESC: Mouse Embryonic Stem Cells \\ Klf4: Kruppel-Like Factor 4 \\ MEFs: Mouse Embryonic Fibroblasts \\ MHC: Myosin Heavy Chain \\ Oct4: POU Domain, Class 5, Transcription Factor 1 \\ Pax6: $\quad$ Paired Box Protein 6 \\ PBS: Phosphate-Buffered Saline \\ PCR: Polymerase Chain Reaction \\ RT: Reverse Transcription \\ SB: $\quad$ Sleeping Beauty Transposon/Transposase \\ Sox2: $\quad$ SRY (sex determining region Y)-box 2 \\ Sox17: SRY (sex determining region Y)-box 17 \\ c-myc: V-Myc Avian Myelocytomatosis Viral Oncogene Homolog \\ UTF1: Undifferentiated Embryonic Cell Transcription Factor 1
}

CAG: Synthetic Promoter containing the (C) cytomegalovirus early enhancer element, (A) the promoter, the first exon and the first intron of chicken beta-actin gene, and $(\mathrm{G})$ the splice acceptor of the rabbit beta-globin gene

\title{
Potential Conflicts of Interests
}

None

\section{Acknowledgments}

Dr. Wilfried Kues (Institute of Farm Animal Genetics, Friedrich-Loeffler-Institute, Mariensee, Neustadt, Germany) for the injection of the immunodefficient mice. Dr. Hans Schoeler (Max-Planck-Institute for Molecular Biomedicine, Muenster, Germany) for providing the OG2 reporter mice. Dr. Zoltan Ivics (Paul-Ehrlich Institute, Langen, Germany) for providing the SB transposon backbone and the SB transposase expression plasmid. Dr. Poul Hyttel (University of Copenhagen, Copenhagen, Denmark) for allowing us to use and modify the transposon plasmid which was generated by Dr. Stoyan Petkov in his laboratory. 
Non-silenced heterologous transgenes in miPSC

\section{Sponsor / Grants}

German Research Foundation (Deutsche Forschungsgemeinschaft), Grant Ni 256/ 32-1.

\section{Additional Information}

Supplementary Information accompanies this article. Supplementary figures, Video and table are linked to the online version of the article.

\section{Corresponding Authors}

1. Stoyan Petkov, German Primate Center, Kellnerweg 4,37077 Göttingen, Germany; Email: spetkov@ dpz.eu

2. Heiner Niemann, Institute for Farm Animal Genetics, Friedrich-Loeffler-Institute, Hoeltystrasse 10, 31535 Neustadt am Ruebenberge, Germany; Email: heiner.niemann@fli.bund.de 